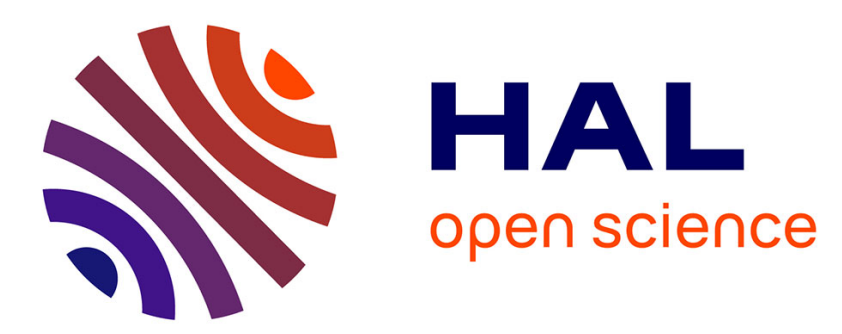

\title{
The Hidden Conformation of Human Histo-blood Group Antigen is a Determinant for Recognition by Pathogen Lectins
}

Jérémie Topin, Mickaël Lelimousin, Julie Arnaud, Aymeric Audfray, Serge Perez, Annabelle Varrot, Anne Imberty

\section{To cite this version:}

Jérémie Topin, Mickaël Lelimousin, Julie Arnaud, Aymeric Audfray, Serge Perez, et al.. The Hidden Conformation of Human Histo-blood Group Antigen is a Determinant for Recognition by Pathogen Lectins. ACS Chemical Biology, 2016, 11 (7), pp.2011-2020. 10.1021/acschembio.6b00333 . hal02381875

\section{HAL Id: hal-02381875 \\ https://hal.science/hal-02381875}

Submitted on 26 Nov 2019

HAL is a multi-disciplinary open access archive for the deposit and dissemination of scientific research documents, whether they are published or not. The documents may come from teaching and research institutions in France or abroad, or from public or private research centers.
L'archive ouverte pluridisciplinaire HAL, est destinée au dépôt et à la diffusion de documents scientifiques de niveau recherche, publiés ou non, émanant des établissements d'enseignement et de recherche français ou étrangers, des laboratoires publics ou privés. 


\title{
The Hidden Conformation of Human Histo-blood Group Antigen is a Determinant for Recognition by Pathogen Lectins
}

\author{
Jérémie Topin ${ }^{\mathrm{a}}$, Mickaël Lelimousin ${ }^{\mathrm{a}}$, Julie Arnaud ${ }^{\mathrm{a}}$, Aymeric Audfray ${ }^{\mathrm{a}}{ }^{\dagger}$, Serge Pérez ${ }^{\mathrm{b}}$, Annabelle Varrot ${ }^{\mathrm{a}}$, \\ Anne Imberty ${ }^{a *}$ \\ ${ }^{a}$ CERMAV UPR5301, CNRS and Université Grenoble Alpes, BP 53, 38041 Grenoble cedex 9, France. ${ }^{b}$ DPM UMR5063, \\ Université Grenoble Alpes and CNRS, BP 53, 38041 Grenoble cedex 9, France
}

KEYWORDS Oligosaccharide, Flexibility, Blood group antigen, Lectin.

\begin{abstract}
Histo-blood group epitopes are fucosylated branched oligosaccharides with well-defined conformations in solution that are recognized by receptors, such as lectins from pathogens. We report here the results of a series of experimental and computational endeavours revealing the unusual distortion of histo-blood group antigens by bacterial and fungal lectins. The Lewis $\mathrm{x}$ trisaccharide adopts a rigid closed conformation in solution, whilst crystallography and molecular dynamics reveal several higher energy open conformations when bound to the Ralstonia solanacearum lectin, which is in agreement with thermodynamic and kinetic measurements. Extensive molecular dynamics simulations confirm rare transient Le ${ }^{\mathrm{x}}$ openings in solution, frequently assisted by distortion of the central $\mathrm{N}$-acetyl-glucosamine ring. Additional directed molecular dynamic trajectories revealed the role of a conserved tryptophan residue in guiding the fucose into the binding site. Our findings show that conformational adaptation of oligosaccharides is of paramount importance in cell recognition and should be considered when designing anti-infective glyco-compounds.
\end{abstract}

\section{INTRODUCTION}

The current recognition of the importance of protein-glycan recognition in cellular processes ${ }^{1}$ is driving the efforts to elucidate the molecular basis underpinning such processes. Most carbohydrate molecules, also referred to as glycans, are considered to be flexible molecules. However, some of them, such as blood group antigens of the $\mathrm{ABH}(\mathrm{O})$ and Lewis systems (Fig. 1A), have been shown to have a well-defined conformation in solution, due to the presence of one or two fucosylated branches which restrict the number of low energy conformations that can be adopted. ${ }^{2}$ In the quest of unravelling the molecular basis dictating the recognition of carbohydrates by proteins, the present understanding is that the pre-formed conformation in solution is likely to be the bio-active one.

Despite a series of supporting evidence that the bound conformation is that occurring in solution, we raised the question whether such a hypothesis was indeed the paradigm or whether exceptions could be found. The conformations of fucosylated Lewis oligosaccharides, are considered to be rigid in solution, adopting a single shape referred to as the "Closed" conformation. ${ }^{24}$ This rigid shape is due to stacking between fucose (Fuc) and galactose (Gal) rings, by a non-conventional $\mathrm{CH}$... hydrogen bond and by steric hindrance of the $\mathrm{N}$-acetyl group of GlcNAc (Fig. 1B). The crystal structure of Lewis $x\left(\mathrm{Le}^{\mathrm{x}}\right)$ trisaccharide ${ }^{5}$, together with $\mathrm{NMR}^{6-9}$ and modeling data, ${ }^{10,11}$ confirmed that the trisaccharide presents only limited conformational fluctuations around the Closed shape.
Since $\operatorname{Le}^{\mathrm{x}}$ and sialyl Lewis $\mathrm{x}\left(\mathrm{SLe}^{\mathrm{x}}\right)$ are key players in many pathologies related to inflammation, cancer and infection ${ }^{4,12}$ and in order to understand and hence manipulate the binding process, it is fundamental to determine if the Closed conformation is maintained during interaction. Crystal structures of $\mathrm{Le}^{\mathrm{x}}$ and SLe ${ }^{\mathrm{x}}$ complexed with lectins or antibodies show the existence of the Closed conformation in the binding sites. This was also confirmed in solution by NMR with the lectins DCSIGN ${ }^{13}$ and E-selectin. ${ }^{14}$ There is therefore a consensus for all carbohydrate-binding proteins, except for a family of fungal and bacterial lectins corresponding to the fucose-binding 6-bladed $\beta$ propeller fold. Indeed, distortion of the $\mathrm{Le}^{\mathrm{x}}$ core was reported by an NMR study of SLe ${ }^{x}$ in interaction for the AAL lectin from the mushroom Aleuria aurantia, ${ }^{15}$ this structure being latter the first 6-bladed $\beta$-propeller lectin to be described. ${ }^{16} \mathrm{We}$ then analyzed the conformations of the $\mathrm{ABH}$ and Lewis antigens when bound other $\beta$-propeller lectins from the lung pathogens Burkholderia ambifaria (BambL) $)^{17,18}$ and Aspergillus fumigatus (AFL1) ${ }^{19}$ respectively. While most fucosylated glycans were found in one of their low energy conformations, unexpected distortion of $\mathrm{Le}^{\mathrm{x}}$ was observed in the binding site of both lectins.

We present here a complete evaluation of the conformational behavior of $\mathrm{Le}^{\mathrm{x}}$, both in solution and in protein binding sites. In addition to the analysis of all the available complexes from lectins in the crystalline state, we describe new crystal structures of the RSL lectin from the bacterium Ralstonia solanacearum bound to $\mathrm{Le}^{\mathrm{x}}$ and SLe ${ }^{\mathrm{x}}$. Several "Open" conformations of $\mathrm{Le}^{\mathrm{x}}$ 
were identified in the binding sites of the $\beta$-propeller lectins. We then compared conformational dynamics of $\mathrm{Le}^{\mathrm{x}}$ in solution to that observed in the RSL binding site. Biophysical studies were performed to characterize the thermodynamics and the kinetics of binding to this lectin. Lastly, extended molecular dynamics (MD) simulations shed light on the complete binding pathways. Overall, the present study provides unprecedented knowledge on the conformational adaptation of glycans when interacting with protein receptors. Since a precise understanding of the recognition mechanism is required to design glycoderived compounds of therapeutical interest, ${ }^{20}$ the results herein have enormous potential to guide new approaches to drug design.

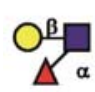

Lewis $\mathrm{x}$

$\left(\operatorname{Le}^{x}\right)$

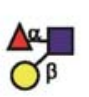

Lewis a $\left(\mathrm{Le}^{\mathrm{a}}\right)$

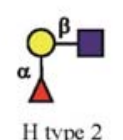

H type 2
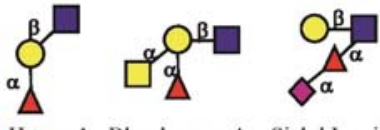

l Lewis $\left(\mathrm{SLe}^{\mathrm{x}}\right)$
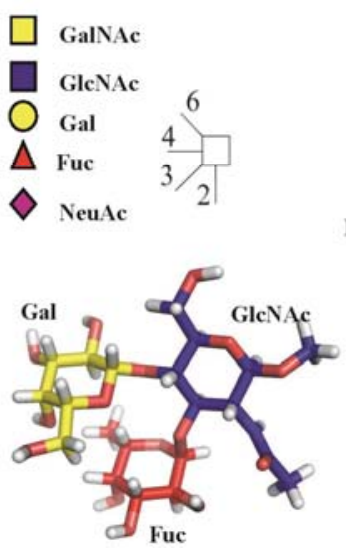

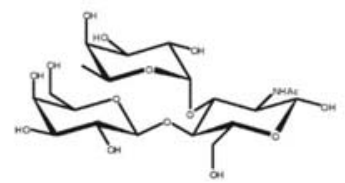

$\mathrm{Le}^{x}: \operatorname{Gal}(\beta 1-4)[\mathrm{Fuc}(\alpha 1-3)] \mathrm{GlcNAc}$

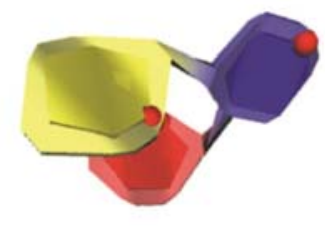

Scheme 1. Schematic representation of selected histo-blood group fucosylated oligosaccharides, together with convention for monosaccharide and linkages representation and different representation of the crystal structure conformation ${ }^{5}$ of $\mathrm{Le}^{\mathrm{x}}$ trisaccharide with use of sticks or SweetUnityMol. ${ }^{21}$

\section{MATERIALS AND METHODS}

Materials. Recombinant RSL was produced in Escherichia coli as previously described ${ }^{22}$ and purified by affinity on mannosesepharose column (Sigma-Aldrich). All oligosaccharides have been obtained from Elicityl (Crolles, France).

Crystallization and structures determination. Crystals of RSL were obtained by the hanging drop vapor diffusion method using $2 \mu \mathrm{l}$ of drops containing a 50:50 (v/v) mix of protein and reservoir solution at $19{ }^{\circ} \mathrm{C}$. The protein at $10 \mathrm{mg} \cdot \mathrm{ml}^{-1}$ in 20 $\mathrm{mM}$ Tris/ $\mathrm{HCl} \mathrm{pH} 7.5$ and $150 \mathrm{mM} \mathrm{NaCl}$ was incubated with 5 $\mathrm{mM}$ of ligand during $1 \mathrm{~h}$ at room temperature prior to cocrystallization. In both cases, the ligand was a tetrasaccharide. For the Lewis X complex, crystals plates were obtained from a solution containing 27\% PEG6K and 0.1 M Tris-HCl pH 8.5. Plates were transferred in a solution were PEG6K concentration was increased to $30 \%$ for cryoprotection prior mounting in a cryoloop and flash-freezed in liquid nitrogen. For the SialylLewis $\mathrm{X}$ complex, rods were obtained from a solution containing $30 \%$ PEG6K and $0.28 \mathrm{M}$ Tris- $\mathrm{HCl} \mathrm{pH} 8.5$ and were directly flash-frozen in a cryoloop. Diffraction data were collected at 100 $\mathrm{K}$ at the European Synchrotron Radiation Facility (Grenoble, France) on beamline ID14-4 using an ADSC Quantum Q315r detector and beamline ID23-2 using a MARCCD detector for the $\mathrm{Le}^{\mathrm{x}}$ and $\mathrm{Sle}^{\mathrm{x}}$ complexes respectively. The data were processed using $\mathrm{XDS}^{23}$. All further computing was performed using the CCP4 suite. ${ }^{24}$ Data quality statistics are summarized in Table S1. The structures were solved by molecular replacement using PHASER ${ }^{25}$ and the trimer coordinates from 2BT9 as search model. Five percent of the observations were set aside for cross-validation analysis, and hydrogen atoms were added in their riding positions and used for geometry and structurefactor calculations. The initial model was optimized with Arp${ }_{\text {wArp }}{ }^{26}$ prior refinement using restrained maximum likelihood refinement in REFMAC $5.8^{27}$ iterated with manual rebuilding in $\mathrm{Coot}^{28}$. Incorporation of the ligand was performed after inspection of the 2Fo-DFc weighted maps. Water molecules, introduced automatically using Coot, were inspected manually. The quality of the models was assessed using the PDB validation server (http://wwpdb-validation.wwpdb.org/validservice/) and coordinates were deposited in the Protein Data Bank under code 5ajb and 5 ajc for the $\mathrm{Le}^{\mathrm{x}}$ and $\mathrm{Sle}^{\mathrm{x}}$ complexes respectively.

Titration microcalorimetry. Recombinant lyophilized RSL was dissolved in buffer $(20 \mathrm{mM}$ Tris $/ \mathrm{HCl} \mathrm{pH} \mathrm{7.5,} \mathrm{NaCl} 150 \mathrm{mM}$ with $0.03 \mathrm{mM} \mathrm{CaCl}_{2}$ ). Oligosaccharide ligands were dissolved in the same buffer, and loaded in the injection syringe. ITC was performed with an ITC200 microcalorimeter (MicroCal Inc.) at $25^{\circ} \mathrm{C}$. Titration was performed with 20 of $2 \mu \mathrm{l}$ injections of carbohydrate ligands ( 1.3 to $2.0 \mathrm{mM}$ ) every $300 \mathrm{~s}$ in the lectin containing cell. Data were fitted with MicroCal Origin 7 software, according to standard procedures. Fitted data yielded the stoichiometry $(n)$, the association contant $\left(K_{a}\right)$ and the enthalpy of binding $(\Delta \mathrm{H})$. Other thermodynamic parameters (i.e. changes in free energy, $\Delta \mathrm{G}$, and entropy, $\Delta \mathrm{S}$ ) were calculated from the equation $\Delta \mathrm{G}=\Delta \mathrm{H}-\mathrm{T} \Delta \mathrm{S}=-\mathrm{RT} \ln \mathrm{K}_{\mathrm{a}}$ in which $\mathrm{T}$ is the absolute temperature and $\mathrm{R}=8.314 \mathrm{~J} \mathrm{~mol}^{-1} \mathrm{~K}^{-1}$. Two independent titrations were performed for each ligand tested. ITC figures were prepared using the Origin software provided with the apparatus, or with NITPIC ${ }^{29}$ and Gussi. ${ }^{30}$

Surface Plasmon Resonance. SPR experiments were performed on a Biacore X100 instrument (GE Healthcare) at $25^{\circ} \mathrm{C}$ in HBS (10 mM Hepes/ $\mathrm{NaOH}, \mathrm{pH} 7.5,150 \mathrm{mM} \mathrm{NaCl}, 0.05 \%$ Tween 20 and $3 \mathrm{mM}$ EDTA) at a flow rate of $30 \mu \mathrm{min}^{-1} .354$ resonance units of RSL were immobilized on a research grade CM5 chip (channel 2, $2 \mu \mathrm{g} \mathrm{ml}^{-1}$ ) using amine coupling procedure; channel 1 was used as control. Experiments consisted of injection (association $180 \mathrm{~s}$, dissociation $180 \mathrm{~s}$ ) of decreasing concentration of $\mathrm{Le}^{\mathrm{x}}$ tetrasaccharide (2-fold cascade dilutions from 200 to $0.4 \mu \mathrm{M})$ and $\mathrm{H}$ type 2 tetrasaccharide $(50$ to $0.05 \mu \mathrm{M})$. The chip was fully regenerated by two injections of $1 \mathrm{M}$ fucose in running buffer for $80 \mathrm{~s}$. Binding was measured as resonance units over time after blank subtraction, and data were then evaluated by using the Biacore X100 evaluation software, version 2.0.

Molecular dynamics. Molecular dynamics simulations were carried out using the AMBER12 program $^{31}$ in the isotherm isobar thermodynamic ensemble at $300 \mathrm{~K}$, using the ff99SB force-field parameters for protein ${ }^{32}$ and GLYCAM06 for saccha- 
rides $^{33}$. Simulations were performed with the pmemd.cuda module using SHAKE algorithm on bonds involving hydrogen atoms. A time step of 2 fs was applied. Particle Mesh Ewald (PME) was used to handle long-range electrostatics interaction. The cut-off for non-bonded van der Waals interactions was set to $8 \AA$. The temperature and the pressure were kept constant using a Langevin thermostat with a collision frequency of $2 \mathrm{ps}^{-1}$ and a weak coupling anisotropic algorithm with a relaxation time of $2 \mathrm{ps}^{-1}$, respectively. The system was optimized in a stepwise manner. 10000 steps of minimization with 5000 steps in conjugate gradient were run with restraints of $20 \mathrm{kcal} \cdot \mathrm{mol}^{-1} \cdot \AA^{-2}$ applied on all atoms of the complex. The minimization was then followed by 20 ps of molecular dynamics equilibration (NVT, 100K) with the same restraints. Restraints were then reduced by $5 \mathrm{kcal} \cdot \mathrm{mol}^{-1} \cdot \AA^{-2}$ and another cycle of minimizationequilibration was made. Finally, the system was minimized for 10000 steps without applying any restraints. The system was then heated from 100 to $300 \mathrm{~K}$ using a Langevin thermostat with a collision frequency of $5 \mathrm{ps}^{-1}$. Initial velocities were derived from a Maxwellian distribution at $100 \mathrm{~K}$ using a random seed for each simulation. 10 ns equilibration phase in the NVT and NPT ensemble were then run. Finally production phase was performed in the NPT ensemble at $300 \mathrm{~K}$.

Molecular dynamics simulations of $L e^{x}$ in RSL. The crystal structure of RSL in trimeric state was considered for calculation therefore generating for each simulation three trajectories of $\mathrm{Le}^{\mathrm{x}}$ in the inter-monomeric binding site and three in the intra binding site. In order to enhance sampling, two independents simulations were carrying out with different starting conformations of the glycan for 900 ns, resulting in 12 independent trajectories of $\mathrm{Le}^{\mathrm{x}}$. The protonation state of each residue was assigned using $\mathrm{H}^{++}$web server ( http://biophysics.cs.vt.edu/ $\mathrm{H}^{++}$), ${ }^{34,35}$ resulting in a neutral charge of the protein. The only histidine residue (His60) was protonated on $\mathrm{N}_{\varepsilon}$ atom. The water phase was extended to a distance of $10 \AA$ from any solute atom using a TI3P water model. The system was optimized following the protocol previously described. Finally a production phase of 900 ns was carried out in NPT ensemble. In order to test the robustness of the results, we performed an additional $1 \mu$ s-long plain MD simulation starting from the crystal structure of RSL in complex with $\mathrm{Le}^{\mathrm{x}}$ (pdb id: 5AJB), using the recent AMBER-ff14SB ${ }^{36}$ and the GLYCAM06-j force fields together with similar simulation parameters were used than those previously described. The $\mathrm{Le}^{\mathrm{x}}$ molecules were considered in their conformation observed in the crystal structure, with one empty binding site.

Molecular dynamics simulations of $\mathrm{Le}^{x}$ in water. Three distinct initial conformations of the $\mathrm{Le}^{\mathrm{x}}$ trisaccharide were selected to study its behaviour in water phase: Open I, Open III and Closed. In order to enhance the conformational sampling, 10 replicate MD simulations were carried out. For each structure, a random seed was used for velocity generation during the heating phase.

Simulating the unbinding $L e^{x}$ to RSL. An Umbrella Sampling (US) protocol was applied to simulate $\mathrm{Le}^{\mathrm{x}}$ unbinding event. Starting from a previously equilibrated structure of the complex RSL-Le ${ }^{\mathrm{x}}$ a bias was applied between the $\mathrm{C} 3$ atom from fucose ring and the $\mathrm{C}$ atom from the Arg61 residue. The distance between the

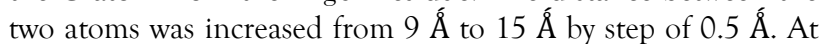
each step, the distance was constrained with a harmonic poten- tial and a force constant of $7 \mathrm{kcal} \cdot \mathrm{mol}^{-1} \cdot \AA^{-2}$. For each window an equilibration phase of $2 \mathrm{~ns}$ was run before a production of 20 ns, which led to a total simulation time of 396 ns The potential of mean force (PMF) was calculated using WHAM software $^{37}$ and standard deviation were calculated by bootstrapping analysis.

Simulating the binding $L e^{x}$ to RSL. In a first attempt to model the binding event, a $1 \mu$ s-long plain MD simulation was performed starting from the apo-form of RSL surrounded by $16 \mathrm{Le}^{\mathrm{x}}$ molecules in solution. The AMBER-ff14SB and GLYCAM06-j force fields were used. The initial simulation box was built with 27 solvated $\mathrm{Le}^{\mathrm{x}}$ molecules periodically distributed, and then molecules in steric clashes with the centred protein were removed. Analysis of contact between the glycans and the protein surfaces has been performed. We finally performed four targeted molecular dynamic (tMD) simulations ${ }^{38}$ to investigate the whole binding mechanism of $\mathrm{Le}^{\mathrm{x}}$ into RSL binding sites, selecting two intra-monomeric and two inter-monomeric binding sites. The additional computed tMD force in comparison to a plain MD framework corresponded to the following energy term:

$\mathrm{E}_{\mathrm{t} M \mathrm{D}}=(\mathrm{k} / 2) \cdot \mathrm{N} \cdot(\mathrm{rmsd})^{2}$

where $\mathrm{k}$ is a force constant used to adjust the magnitude of the tMD force, $\mathrm{N}$ is a number of atoms selected for the calculation of the rmsd, and the rmsd is the mass-weighted root mean square displacement of the selected atoms in comparison to a reference structure. The reference structure was here the crystal structure of the RSL- Le ${ }^{x}$ complex. As starting configuration of each simulation, a $\mathrm{Le}^{\mathrm{x}}$ molecule was located in a random orientation at a distance of ${ }^{\sim} 10 \AA$ from the RSL binding site. We used a weak force constant of $0.1 \mathrm{kcal} \mathrm{mol}^{-1} \AA^{-2}$ in order to minimize the bias from the tMD framework. A total of 58 atoms were picked up for the calculation of the rmsd, namely the non-hydrogen atoms of the two tryptophans, the glutamate and the arginine of the binding site and those of the fucose ring of $\mathrm{Le}^{\mathrm{x}}$. In this way, the tMD force did not affect the $\mathrm{Le}^{\mathrm{x}}$ conformation. We note that at each step, for the calculation of the rmsd, the system was aligned on the reference structure along the backbone atoms of the protein. The AMBER-ff14SB and GLYCAM06-j force fields were used.

\section{RESULTS AND DISCUSSION}

The $\beta$-propeller lectin from $R$. solanacearum binds $\mathrm{Le}^{\mathrm{x}}$ in several Open conformations. Crystals of RSL complexed with $\mathrm{Le}^{\mathrm{x}}$ and SLe ${ }^{\mathrm{x}}$ tetrasaccharides have been obtained by cocrystallization and diffracted to 1.8 and $1.7 \AA$ resolution, respectively (see Supplementary Table 1 for statistics). The content of the asymmetric unit is a $\beta$-propeller consisting of three peptidic chains (A, B and $\mathrm{C}$ ) and six binding sites (intra and inter monomeric) (Fig. 2A). The 3D-structure of the protein and of the fucose binding sites are in agreement with previously reported data $^{22}$. In all sites, the fucose residue establishes hydrogen bonds to Arg, Glu, Ala and Trp residues while its methyl group locates in an hydrophobic pocket made by Trp and Ile residues.

The quality of the electron density maps allowed the location of the entire $\mathrm{Le}^{\mathrm{x}}$ core trisaccharide in five of the binding sites of $\mathrm{RSL} / \mathrm{Le}^{\mathrm{x}}$ and two of RSL/SLe ${ }^{\mathrm{x}}$ complexes. The whole SLe ${ }^{\mathrm{x}}$ tetrasaccharide is clearly observed in one site only (Supplementary Fig. 1). While the fucose is always located with the same 
orientation in the binding site, the $\mathrm{Le}^{\mathrm{x}}$ core adopts three very different conformations, resulting in different contacts between the oligosaccharide and the protein (Fig. 2B). The conformation labelled "Open I" is observed in site A of both complexes; it presents an hydrogen bond between $\mathrm{N}$-acetyl group of GlcNAc and Arg17, and only one water bridged contact for the galactose moiety. Conformation Open II is observed in site B of the complex with $\mathrm{Le}^{\mathrm{x}}$; it brings the galactose close to the protein surface with several hydrogen bonds in particular to Trp10 and Asp32. Conformation Open III is observed in the three intermonomeric sites of RSL/Le ${ }^{x}$ complex; the Gal and GlcNAc residues do not interact directly with the protein but are involved in a very extended hydrogen bonds network with water molecules bridging to the protein surface.
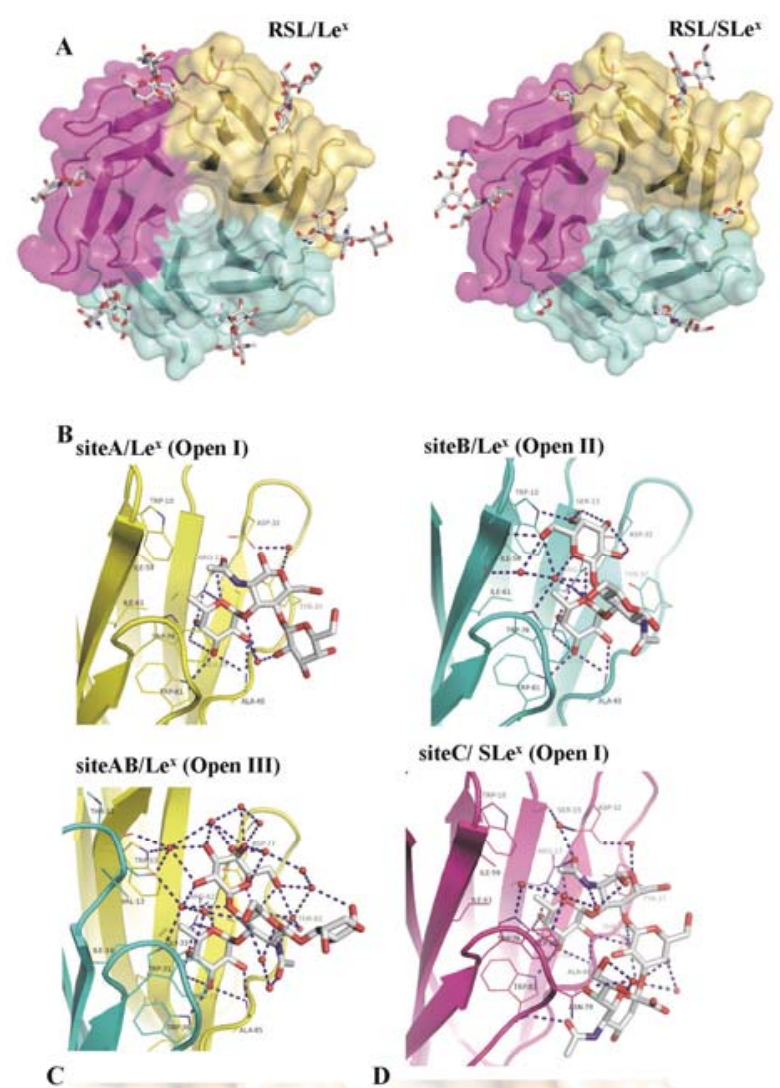

C
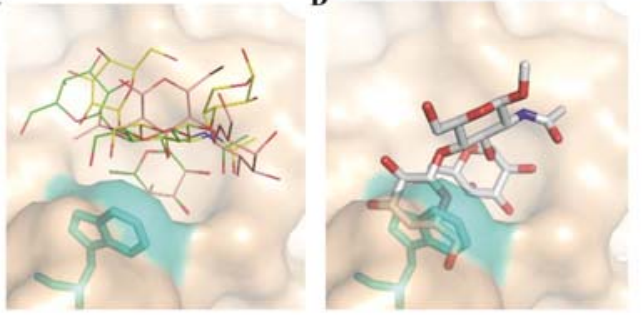

Figure 2. Crystal structures of RSL complexed with $\mathrm{Le}^{\mathrm{x}}$ and SLe ${ }^{\mathrm{x}}$. A. The oligosaccharides are represented by stick models and the proteins with surfaces of different colors coding for each monomer. B. Details of the hydrogen bond network in different binding sites of the complexes. The hydrogen bonds are represented by blue dashed lines and waters as red spheres. C. Superimposition of the three conformations of $\mathrm{Le}^{\mathrm{x}}$ observed in RSL binding sites (green: Open I in site A, pink: Open II in site B, and yellow: Open III in site AB). Trp 76 has been colored in cyan. D. Superimposition of the Closed conformation of $\mathrm{Le}^{\mathrm{x}}$ on the fucose in RSL binding site demonstrating the resulting steric clash between galactose and Trp76.

When the three conformations of the $\mathrm{Le}^{\mathrm{x}}$ trisaccharide core are superimposed on their fucose ring in the RSL binding site, they span different regions of the large pocket above the primary binding site (Fig. 2C). All three conformations differ from the rigid Closed shape previously described. When the Closed Le ${ }^{x}$ conformation is docked in the RSL site by positioning the fucose in its canonical position, this generates a strong steric clash between the galactose residue and $\operatorname{Trp} 76$ or $\operatorname{Trp} 31$ in intra- and inter-monomeric sites, respectively (Fig. 2D). As a Trp residue stacks to the fucose in all binding sites and as it is conserved in all lectins of the same $\beta$-propeller family, it may play a major role in the occurrence of the non-canonical Open conformations of $\mathrm{Le}^{\mathrm{x}}$.

Only $\beta$-propeller lectins are able to open the solution conformation of Lewis oligosaccharides. The conformational analysis of $\mathrm{Le}^{\mathrm{x}}$ in protein binding sites was first applied to the other fucose-specific $\beta$-propeller lectins for which crystal structures are available. Crystals of AFL have been obtained as a complex with Lewis Y ( $\mathrm{Le}^{\mathrm{y}}$ - a tetrasaccharide with two fucose residues) (code 4D4U) ${ }^{39}$. A new shape of the Lewis oligosaccharide, referred to as Open IV, is observed in two of the binding sites. The BambL/Le ${ }^{x}$ complex (code $\left.3 Z W W 1\right)^{18}$ presents electron density for oligosaccharides in two sites; one corresponds to the Open III conformation and the other to a different one with distortion of GlcNAc ring in skew-boat ${ }^{\circ} \mathrm{S}_{2}$, which is referred as Open $\mathrm{V}$. All of these observed conformations are displayed in Figure $3 \mathrm{~A}$ using SweetUnityMol ${ }^{21}$ for clearer representation of the different shapes.

Oligosaccharides conformations are primarily defined by the relative orientations of their constituting monosaccharides at their glycosidic linkages. When reporting the values of torsion angles at each linkage on the corresponding potential energy map previously calculated with MM3 force-field ${ }^{11}$ (Fig. 3B), the Closed shape falls into the two main low energy regions of both $\alpha$ Fuc1-3GlcNAc and $\beta$ Gal1-4GlcNAc maps. On the opposite, all of the open conformations have at least one of their glycosidic linkage conformation lying in secondary minima. When compared to the canonical Closed conformation observed in solution, Open I is characterized by a large change of angle for both $\alpha$ Fuc1-3GlcNAc and $\beta$ Gal1-4GlcNAc linkages, whereas Open II and Open III are mostly distorted about the $\beta$ Gal14GlcNAc one. Open IV exhibits variation for both $\Phi$ and Yangles at the $\alpha$ Fuc1-3GlcNAc linkage. Open V is not represented on the map as its GlcNAc ring is distorted, a situation that was not considered while calculating these potential energy maps.

In order to simplify the description of the different shapes, a 2D-representation was created based on the relative distance and orientation between the fucose and galactose residues. A polar coordinate system (or radar graph) has been designed with the radius $\mathrm{r}$ as the distance between $\mathrm{C}_{\text {Fuc }}$ and $\mathrm{O} 4_{\mathrm{Gal}}$ atoms and the polar angle $\Theta$ representing the dihedral angle between fucose and galactose ring planes (Fig. 3C). This representation enables a clear discrimination between the different shapes observed in the crystal structures. The Closed conformation with stacked galactose and fucose rings is characterized by a 
small value of $\mathrm{r}(<5 \AA)$ and a dihedral angle $\Theta$ close to $0^{\circ}$, whereas-all open conformations brings the two rings farther apart.
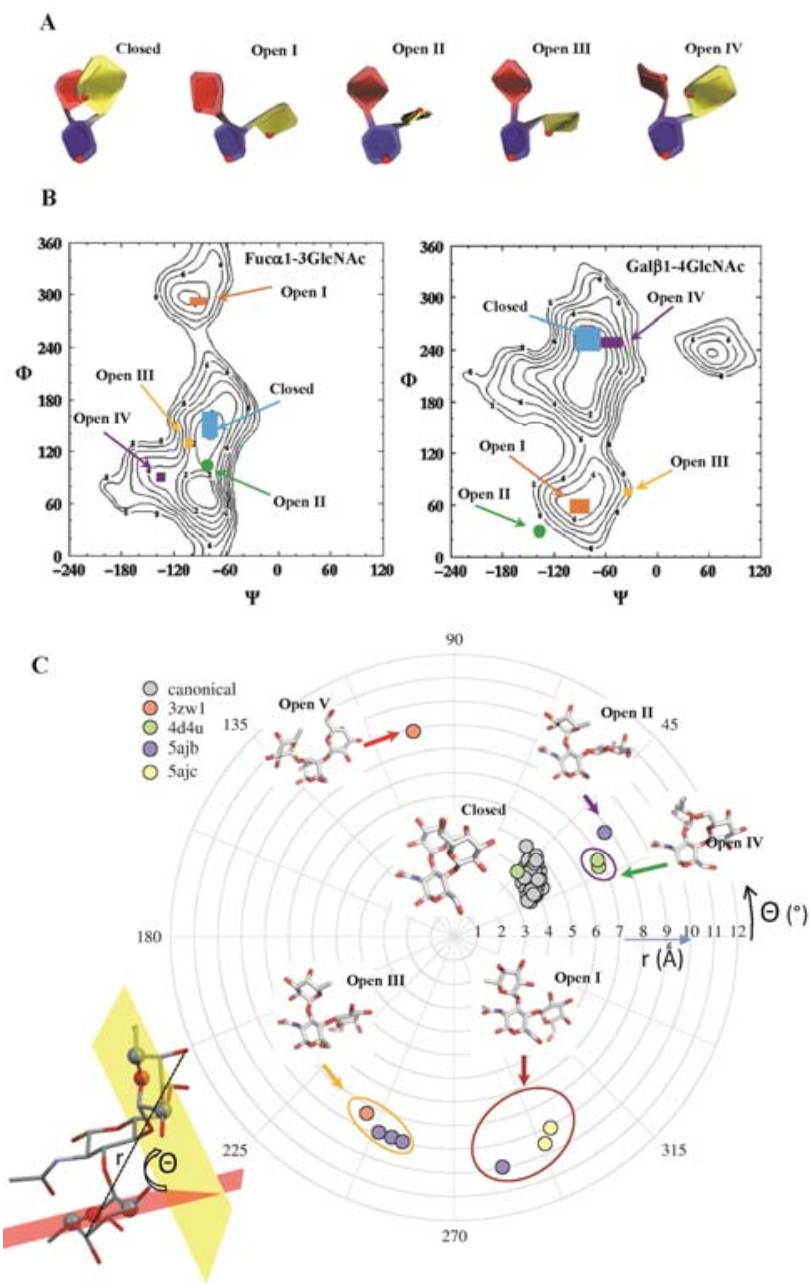

Figure 3. Analysis of the 60 conformations of Le moiety from 30 crystal structures of protein complexes at resolution better than 2.5 $\AA$ (see Supplementary Table 2 and Table 3). A. Sweet-unity representation of the different shapes observed with same color coding as used in Fig. 1. B. Analysis of glycosidic linkages conformation as a function of the torsion angles $\Phi_{(\mathrm{O} 5-\mathrm{Cl}-\mathrm{O} 1-\mathrm{CX})}$ and $\Psi_{(\mathrm{Cl} 1-\mathrm{Ol}-\mathrm{CX}-\mathrm{CX}+1)}$ and superimposition on the MM3 energy map of each disaccharide ${ }^{11}$. C. Analysis of the shape of the observed conformations using a polar coordinate system graph with the radius $r(\AA)$ representing the distance between $\mathrm{C} 4_{\text {Fuc }}$ and $\mathrm{O} 4_{\mathrm{Gal}}$ atoms and the polar angle $\Theta\left(^{\circ}\right)$ representing the dihedral angle between fucose and galactose ring planes defined by $\mathrm{C} 2_{\mathrm{Gal}}-\mathrm{C} 5_{\mathrm{Gal}}-\mathrm{O} 5_{\mathrm{Gal}}$ and $\mathrm{O} 5_{\mathrm{Fuc}}-\mathrm{C} 5_{\mathrm{Fuc}} \mathrm{C} 2_{\mathrm{Fuc}}$, respectively.

The analysis of crystal structures of proteins complexed with $\mathrm{Le}^{\mathrm{x}}$ or Le ${ }^{x}$-containing glycans $\left(\mathrm{Le}^{\mathrm{y}}, \mathrm{SLe}^{\mathrm{x}}\right.$ and sulfo $\left.\mathrm{Le}^{\mathrm{x}}\right)$ was extended to all lectins and antibodies, by searching the 3D-Lectin and MAbs databases available in Glyco3D ${ }^{40}$ (http://glyco3d.cermav.cnrs.fr), and taking into account only the structures with a resolution better than $2.5 \AA$. Including the three $\beta$-propeller lectins described above, the search resulted in 30 crystal structures (Supplementary Table 2) of lectins and antibodies originating from animals, plants, fungi, bacteria and virus. 60 oligosaccharides could be analyzed from these crystal structures (Supplementary Table 3) and for 48 of them the $\operatorname{Le}^{\mathrm{x}}$ moiety was found to adopt the Closed conformation as represented in Figure 3C. The Open conformations are strictly restricted to the $\beta$-propeller family, and all $\beta$-propeller binding sites contain an Open conformation, except for one site of AFL1 complexed with Le ${ }^{y}$. In this particular case, the second fucose of the tetrasaccharide ( $\alpha$ Fuc1-2Gal, and not $\alpha$ Fuc1$3 \mathrm{GlcNAc}$ ) is engaged in the binding site. This analyze confirms that all proteins except the $\beta$-propellers bind $\mathrm{Le}^{\mathrm{x}}$ in its Closed conformation, and that only the binding site of fucose-specific $\beta$-propellers, with stacking Trp residue, is able to select or to induce the Open conformations that are specifically observed for this family of lectins.

Molecular dynamics demonstrates drastic differences in the conformational behavior of $\mathrm{Le}^{\mathrm{x}}$ conformations from solution to RSL binding sites. In order to analyze the conformational dynamics of the $\mathrm{Le}^{\mathrm{x}}$ trisaccharide bound to RSL, the crystal structure of the complex was submitted to two MD simulations with explicit hydration using the AMBER12 program $^{31}$ with the ff99SB force field parameters for protein ${ }^{32}$ and GLYCAM06 for saccharides $^{33}$ (Fig. 4A and Supplementary Fig. 2). Simulations were started either from conformation Open III or Open I in all sites, with duration of 1 and $0.85 \mu$ s, respectively. During the simulations, no change was observed for the location of the fucose in the primary binding site (Supplementary Fig. 3), hence confirming the stability of the hydrogen bond network. On the opposite, GlcNAc and Gal residues displayed large fluctuations exploring at least two among the five Open conformations. These fluctuations were often correlated with variation of the GlcNAc ring pucker away from the ${ }^{4} \mathrm{C}_{1}$ as followed by analysis of three intra-ring torsion angles ${ }^{41}$ (Supplementary Fig. 3). Therefore the variety of conformations observed in the crystal structures truly reflects the flexibility of the trisaccharide in the binding site. This conformational dynamics was also confirmed using the most recent force field AMBER-ff $14 \mathrm{SB}^{36}$ with Glycam06-j (Supplementary Fig. 4).

MD simulations were also performed for $\mathrm{Le}^{\mathrm{x}}$ in solution for 30 independent trajectories of 1 to $10 \mu$ s, starting either from the canonical Closed conformation, or from two of the Open conformations observed in RSL binding sites (Fig. 4B and Supplementary Fig. 5). For the shorter simulation $(1 \mu \mathrm{s})$ starting from the Closed shape, no conformational change was observed. When the simulation started from an Open state, the conformation went to the Closed one after less than $0.2 \mu$ s and remained then stable. However, 5 simulations among the set of 30 displayed short rearrangements from Closed to Open conformations (on a ns timescale) and only one of them showed a longer stay of $0.2 \mu \mathrm{s}$ in the open state with a sampling of different shapes (Fig. 4B). Interestingly, a twist of the GlcNAc ring was observed in most of the opening events. Overall our data show that opening of the $\mathrm{Le}^{\mathrm{x}}$ trisaccharide can also occurs in solution, but as a rare event that can be detected providing that multiple long MD simulations are performed. Such an occurrence may nevertheless be too rare to be detected by NMR methods. 

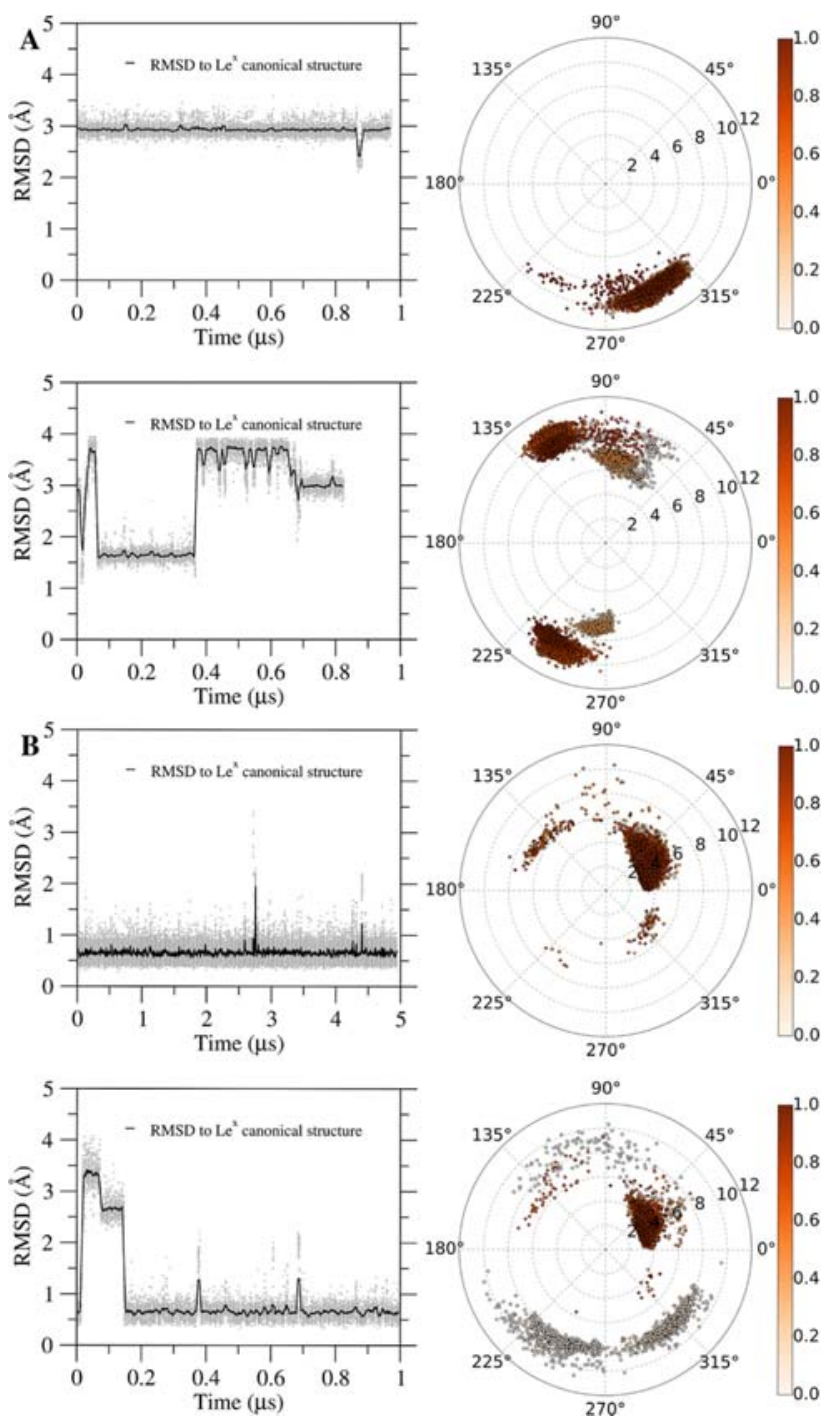

Figure 4. Selected MD trajectories of $\mathrm{Le}^{\mathrm{x}}$. A: Two trajectories of $\mathrm{Le}^{\mathrm{x}}$ in RSL binding site C (top) and AB (bottom). B: Two trajectories of $\mathrm{Le}^{\mathrm{x}}$ in water. Left panel: Time evolution of rmsd $(\AA)$ from canonical Closed structure of $\mathrm{Le}^{\mathrm{x}}$ (grey dots represent the observed values, while the black line reports the running average of the individual values). Right panel: Time evolution of $\mathrm{Le}^{\mathrm{x}}$ shape as the function of fucose-galactose distance $r(\AA)$ and ring-planes angle $\mathrm{Q} \Theta$ $\left(\right.$ in $^{\circ}$ ) defined as in Fig.3 (color coding as relative evolution of time in trajectory). All trajectories are available in supp. info.

Experiments demonstrate conformational enthalpy cost and shorter residence time for $\mathrm{Le}^{\mathrm{x}}$ in RSL binding site when compared to linear oligosaccharides. In order to get experimental data on the binding mechanisms, the thermodynamics and kinetics of RSL binding to several oligosaccharides were investigated through isothermal titration micro-calorimetry (ITC) and surface plasmon resonance (SPR). The titration thermogram as displayed in Figure 5A, is in agreement with the binding of two $\mathrm{Le}^{\mathrm{x}}$ molecules per RSL monomer, and it exhibits the classical exothermic peaks, a characteristic feature of enthalpy-driven interactions (Table 1). The affinity for RLS is in the medium range $\left(K_{d}=35.7 \mu \mathrm{M}\right)$ and comparison with other fucosecontaining glycans (Supplementary Fig. 6) indicates a higher affinity for 2'fucosyllactose ( $\mathrm{H}$ type 5 epitope) and 2'- fucosyllactosamine ( $\mathrm{H}$ type 2 epitope). From previous structural studies of Fuc1-2Gal-containing oligosaccharides (H type series) complexed with RSL or with the related $\mathrm{BambL}^{17,22}$, no deviation from low energy conformation is observed upon binding for these two oligosaccharides. The lower affinity of $\mathrm{Le}^{\mathrm{x}}$ is mostly due to a less negative enthalpy of binding, whereas the entropy change is in the same order of magnitude than those measured for other oligosaccharides. From the thermodynamic analysis, the high-energy Open conformations adopted by Le ${ }^{\mathrm{x}}$ in the binding site is reflected by an enthalpy cost, resulting therefore in a relatively lower affinity, when compared to linear unconstrained oligosaccharides.
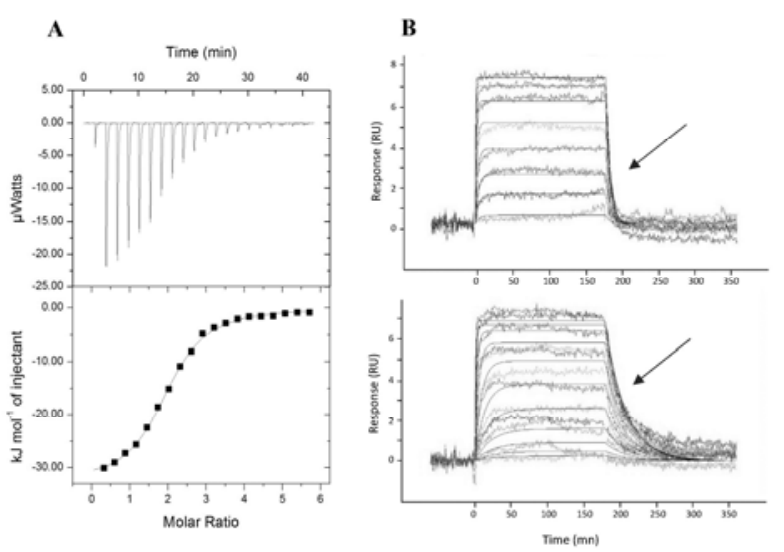

Figure 5. A. Titration calorimetry of $\mathrm{Le}^{\mathrm{x}}$ tetrasaccharide $(5 \mathrm{mM})$ in cell containing $\mathrm{RSL}(172 \mu \mathrm{M})$ at $25^{\circ} \mathrm{C}$ with thermogram resulting from injection and fit of integrated heat. B. Comparison of SPR sensorgrams when circulating $\mathrm{Le}^{\mathrm{x}}$ tetrasaccharide (top) and $\mathrm{H}$ type 2 tetrasaccharide (bottom) at various concentrations on a RSLfunctionnalized chip. The arrow indicates the slower dissociation phase for $\mathrm{H}$ type 2 compared to $\mathrm{Le}^{\mathrm{x}}$.

Table 1. Thermodynamics data. Standard deviations on measured values $\left(\mathrm{K}_{\mathrm{d}}\right.$ and $\left.\Delta \mathrm{H}\right)$ are below $10 \%$.

\begin{tabular}{ccccc}
\cline { 2 - 5 } & $\begin{array}{c}\mathrm{K}_{\mathrm{d}} \\
\mu \mathrm{M}\end{array}$ & $\begin{array}{c}\Delta \mathrm{G} \\
\mathrm{kJ} / \mathrm{mol}\end{array}$ & $\begin{array}{c}\Delta \mathrm{H} \\
\mathrm{kJ} / \mathrm{mol}\end{array}$ & $\begin{array}{c}\mathrm{T} \Delta \mathrm{S} \\
\mathrm{kJ} / \mathrm{mol}\end{array}$ \\
\hline Le $^{\mathrm{x}}{ }_{\text {terra }}$ & 25.7 & -26.2 & -31.1 & -4.9 \\
Le $^{\mathrm{a}}{ }_{\text {tetra }}$ & 18.8 & -27.0 & -29.4 & -2.4 \\
$\mathrm{Le}_{\text {penta }}^{\mathrm{y}}$ & 7.3 & -29.3 & -35.4 & -6.1 \\
$\mathrm{SLe}^{\mathrm{x}}{ }_{\text {penta }}$ & 58.0 & -24.2 & -19.6 & 4.6 \\
$\mathrm{H}$ type $1_{\text {tetra }}$ & 13.8 & -27.7 & -29.8 & -2.1 \\
$\mathrm{H}$ type $2_{\text {tetra }}$ & 4.2 & -30.7 & -38.2 & -7.5 \\
$\mathrm{H}$ type $5_{\text {tri }}{ }^{\mathrm{a}}$ & 0.25 & -37.7 & -39.3 & -1.6 \\
A type $1_{\text {tetra }}{ }^{\mathrm{b}}$ & 125 & -22.3 & -28.7 & -6.4 \\
$\mathrm{~B}$ type $2_{\text {penta }} \mathrm{b}$ & 66.7 & -23.8 & -33.6 & -9.8 \\
\hline
\end{tabular}

[a] from ${ }^{22} ;[b] \mathrm{N}$ fixed to 2

SPR was previously performed to investigate the binding of all Lewis and $\mathrm{ABH}$ blood group oligosaccharides to $\mathrm{RSL}^{22}$; the measured affinities were very close to the ones reported in the present study, as measured by ITC. Another series of SPR experiments were performed with a low density RSL chip in order to reach a precise determination of the kinetics of interaction. The $\mathrm{Le}^{\mathrm{x}}$ and $\mathrm{Le}^{\mathrm{a}}$ branched structure could then be compared with $\mathrm{H}$ type 2 tetrasaccharide (Table 2). The resulting affinity 
results were in agreement with the ITC measurements, and with previous literature data ${ }^{22}$, but some differences could be observed when looking at details of kinetics (Fig. 5B). Indeed, the 13 fold affinity increase for $\mathrm{H}$ type 2 tetrasaccharide when compared to $\mathrm{Le}^{\mathrm{x}}$ is mainly due to a slower dissociation rate (6 fold) and in a lesser way to a faster association rate ( 2 fold). Le ${ }^{x}$ appears to bind at the same speed than linear H-type 2 does, but exits much more rapidly from the site. This observation is in agreement with the strained conformation that Lewis oligosaccharides have to adopt in RSL binding site (spring effect). Whereas the present experimental data could bring some information about the process of dissociation between RSL and the constrained $L^{\mathrm{x}}$, it is more difficult to gain insights into the association process.

Table 2. Kinetics data obtained from SPR

\begin{tabular}{cccc}
\cline { 2 - 4 } & $\begin{array}{c}\mathrm{k}_{\text {on }} \\
1 /(\mathrm{M} \mathrm{s})\end{array}$ & $\begin{array}{c}\mathrm{k}_{\text {off }} \\
1 / \mathrm{s}\end{array}$ & $\begin{array}{c}\mathrm{K}_{\mathrm{d}} \\
\mu \mathrm{M}\end{array}$ \\
\hline Le $_{\text {tetra }}^{\mathrm{x}}$ & 12330 & 0.169 & 13.7 \\
Le $_{\text {tetra }}^{\mathrm{a}}$ & 10080 & 0.154 & 15.3 \\
$\mathrm{H}$ type 2 $_{\text {tetra }}$ & 25430 & 0.027 & 1.06 \\
\hline
\end{tabular}

Modeling the association and dissociation processes reveals the conformational rearrangement. In order to determine the mechanism involved in the distortion-associated binding of $\mathrm{Le}^{\mathrm{x}}$ to RSL, simulations were set to follow the exit and entry pathways. The RSL/Le ${ }^{x}$ complexes are very stable and no dissociation was observed for microsecond simulations (Supplementary Fig. S3). A directed MD simulation was therefore performed with Umbrella Sampling approach in order to pull the $\mathrm{Le}^{\mathrm{x}}$ from the site in equilibrium at each step (Fig. 6A and Supplementary Movie 1). The observed motion corresponds to the sliding of the fucose residue along the Trp indole ring, while maintaining contact with methyl group at $\mathrm{C} 6$ and $\mathrm{CH}$ groups at $\mathrm{C} 4$ and $\mathrm{C} 5$. The protein binding site did not display any conformational flexibility, except for a limited motion of the Trp-containing loop, which resulted in a slight opening of the Trp (limited to 2 ). The other residues, Gal and GlcNAc, moved freely, exploring the different Open shapes of the trisaccharide. It was only after 270 ns and after the release of the fucose from the binding site that the $\mathrm{Le}^{\mathrm{x}}$ could reach its Closed conformation. Again we observed that some conformational changes between the different Open conformations were accompanied by significant distortion of the GlcNAc ring.

The first attempts to observe the entry of $\mathrm{Le}^{\mathrm{x}}$ in the binding site were performed throughout standard MD simulation on a system containing the RSL molecule surrounded by $16 \mathrm{Le}^{\mathrm{x}}$ trisaccharides located in solution far from the protein. (Supplementary Fig. 7). No complete binding of Le ${ }^{\mathrm{x}}$ to RSL could be observed during this simulation. In some instances, some trisaccharides came in close contact to the binding site, the fucose moiety establishing stable transient interactions (20-50ns) with the Trp residue. These trisaccharides remained in the Closed conformation and no opening was observed that could have yield to entry in the binding site. Given the low frequency of $\mathrm{Le}^{\mathrm{x}}$ opening in solution, the probability to observe the complete binding process on this timescale was likely to be too low.
In order to trigger the whole binding process, targeted MD simulations were performed by driving the $\mathrm{Le}^{\mathrm{x}}$ trisaccharide in four binding sites. This procedure was successful in all cases but one, with opening of $\mathrm{Le}^{\mathrm{x}}$ as the Trp pushed the Gal away from the Fuc ring (Supplementary Fig. 8). In site A, the trisaccharide remained closed and could not enter completely in the site, while in sites B and AB it could open to adopt shapes Open II and Open IV with fucose stacked to Trp. A two steps trajectory could be observed in site $\mathrm{BC}$ with rapid opening of $\mathrm{Le}^{\mathrm{x}}$, allowing the fucose to establish weak contact with Trp, followed by the sliding motion along the Trp until complete entry in the site (Fig. 6B and Supplementary Movie 2).
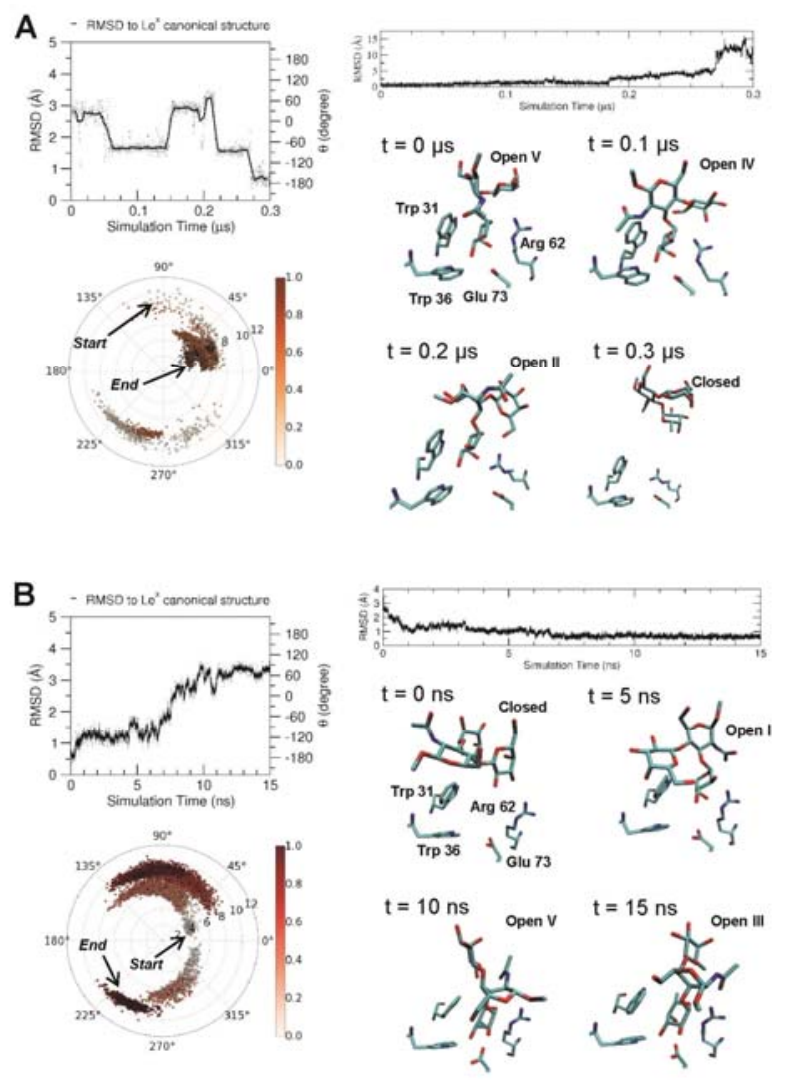

Figure 6. A Simulations of exit and entry of $\operatorname{Le}^{\mathrm{x}}$ in RSL binding site. A. Umbrella sampling simulation of the Le ${ }^{x}$ structure exiting the inter-monomeric $\mathrm{AB}$ binding site of RSL. B. Targeted MD simulation of the $\mathrm{Le}^{\mathrm{x}}$ structure entering the inter-monomeric $\mathrm{BC}$ binding site of RSL. Top-left panels: time evolution of rmsd ( $\mathrm{A})$ from canonical Closed structure of $\mathrm{Le}^{\mathrm{x}}$ (grey dots represent the observed values, while the black line reports the running average of the individual values). Bottom-left panels: time evolution of $\mathrm{Le}^{\mathrm{x}}$ shape as the function of fucose-galactose distance $r(\AA)$ and ringplanes angle $\left({ }^{\circ}\right)$ defined as in Figure 3 (color coding as relative evolution of time in trajectory). Top-right panels: rmsd of the nonhydrogen atoms of both the fucose ring and the binding site (Trp, Arg, Glu) aligned in comparison to the crystal structure of the Le $\mathrm{x}^{\mathrm{x}}$ RSL complex, as a function of the simulation time. Bottom-right panels: Time evolution of the $\mathrm{Le}^{\mathrm{x}}$ structure in vicinity of the RSL binding site. Carbon, nitrogen, oxygen atoms are represented in cyan, blue and red, respectively. For sake of clarity, hydrogens are not shown. 
The entry and exit simulations therefore highlighted the role of the Trp residue in the intermediate stage: the flat indole ring surface of this aromatic amino acid is able to make first van der Waals interaction with the apolar patch on fucose. From this, the opening of the trisaccharide, often accompanied by deformation of GlcNAc, is necessary for complete entering in the binding site.

\section{DISCUSSION AND CONCLUSION}

The rigidity of the $\mathrm{Le}^{\mathrm{x}}$ core in solution is a widely accepted paradigm. However, a recent simulation study, in conjunction with NMR experiments, suggested that the $\mathrm{Le}^{\mathrm{x}}$ core has some flexibility, but is limited to fluctuation of the $\mathrm{N}$-acetyl group orientation coupled with small variations at each glycosidic linkages ${ }^{7}$. The present work therefore reports the first unambiguous experimental evidence from $\mathrm{x}$-ray structure confirmed by computer simulation of the large-range flexibility of $\mathrm{Le}^{\mathrm{x}}$. In solution, the transition events are rare and could be observed only by performing extended MD calculations. From a methodological point of view, this confirms that MD simulations of microsecond timescale are required to decode the conformation of oligosaccharides as previously demonstrated for $\mathrm{N}$-glycan oligosaccharides ${ }^{42}$ and heparan sulfate fragments ${ }^{43}$.

The conformational route that the oligosaccharide could use to change from the closed conformation to an open one is not obvious as the stacking of fucose and galactose limits the flexibility of each linkage. The changes in conformation has to involve either concerted motions at both linkages, or more likely, some distortion of the GlcNAc ring. Of course, the question arises as to whether the frequency of such ring distortions may depend on the parametrization of ring shape in the forcefield. Nevertheless, several studies based on high resolution crystal structures of protein complexes, AFM experiments and simulations have already pointed towards alternative ring puckering besides the ${ }^{4} \mathrm{C}_{1}$ for GlcNAc. ${ }^{42,44}$ Indeed, the distortion of GlcNAc is confirmed, as observed in the crystal structure of $\mathrm{BambL} / \mathrm{Le}^{\mathrm{x}}$ complex ${ }^{18}$ where the ring adopts a ${ }^{\mathrm{O}} \mathrm{S}_{2}$ pucker.

From the ensemble of experimental and theoretical studies described here, it is proposed that a Trp residue of RSL and related lectins plays a crucial role in the stabilization of the open shape of Le $\mathrm{e}^{\mathrm{x}}$ and guides its trajectory into the binding site. Aromatic residues play a key role in protein-carbohydrate interactions, and in particular Trp residues been found to have an increased prevalence of 9 -fold in sugar binding sites. ${ }^{45}$ In the case of RSL, mutants lacking this particular Trp exhibit a loss of 3 orders of magnitude in their affinity towards fucose. ${ }^{46}$ In addition to the stabilization effect, our simulation study suggests that the Trp residue helps to orientate the fucose residue through contact with the hydrophobic patch and that the sliding motion of fucose along the indole ring of Trp assists the opening of the trisaccharide.

The experimental characterization of the interaction indicates that $\mathrm{Le}^{\mathrm{x}}$ binds with lower affinity than linear fucosylated oligosaccharides which do not have to undergo a major conformational change for entering the binding site. In thermodynamic terms, the loss of affinity could be attributed to an enthalpy cost corresponding to the higher energy conformation necessary for binding, whereas in kinetics terms, a faster exit rate is observed, corresponding to the release of the strained conformation.
Lectins are often used as models for characterizing protein/carbohydrate interactions, but most systems used, i.e. plant lectins interacting with human oligosaccharides have no biological significance. In contrast, the present description of the recognition of fucosylated oligosaccharides is highly relevant since these epitopes are present on plant and human tissues and serve as targets for bacterial and fungal lectins. Understanding the conformational behavior of these ligands is therefore a matter of vital scientific interest and will aid the design of high affinity glyco-compounds, or glycomimetics that could compete against the binding of pathogens to host tissues for therapeutic benefit.

\section{ASSOCIATED CONTENT}

Supporting Information. Details about crystal structure, analysis of structures in PDB, molecular modeling, and ITC data are available in supplemental material. "This material is available free of charge via the Internet at http://pubs.acs.org."

\section{AUTHOR INFORMATION}

\section{Corresponding Author}

Anne Imberty: anne.imberty@cermav.cnrs.fr

\section{Present Addresses}

$\dagger$ Present adress: Malvern Instruments, Parc Club du Moulin à Vent, Bât 25, 33 avenue du Docteur Lévy, 69200 Vénissieux, France.

\section{Author Contributions}

The manuscript was written through contributions of all authors. All authors have given approval to the final version of the manuscript.

\section{ACKNOWLEDGMENT}

This work was supported by CNRS, Université Grenoble Alpes, Labex ARCANE (ANR-11-LABX-0003-01) and ERASynbio program SynGlycTis (ANR-14-SYNB-0002-02). The authors are grateful to European Synchrotron Radiation Facility, Grenoble, France for access and technical support to beamlines ID23-2 and ID14-4. MD calculations were perfomed on the CECIC Platform of ICMG. Technical help of Emilie Gillon is warmly appreciated as well as careful reading by Claire Doherty.

\section{ABBREVIATIONS}

RSL, Ralstonia solanacearum lectin; Le $\mathrm{x}^{\mathrm{x}}$ Lewis $\mathrm{x}$ oligosaccharide, $\mathrm{SLe}^{\mathrm{x}}$, sialyl Lewix x oligosaccharide; Le ${ }^{\mathrm{a}}$, Lewis a oligosaccharide; ITC, isothermal titration microcalorimetry; SPR, Surface plasmon resonance; MD, molecular dynamics.

\section{REFERENCES}

(1) Varki, A.; Cummings, R. D.; Esko, J. D.; Freeze, H. H.; Stanley, P.; Bertozzi, C. R.; Hart, G. W.; Etzler, M. E. Essentials of Glycobiology, 2nd edition; Cold Spring Harbor Laboratory Press: Cold Spring Harbor (NY), 2009.

(2) Lemieux, R. U.; Bock, K.; Delbaere, L. T. J.; Koto, S.; Rao, V. S. R. Can. J. Chem. 1980, 58, 631.

(3) Imberty, A.; Breton, C.; Oriol, R.; Mollicone, R.; Pérez, S. Adv. Macromol. Carbohydr. Res. 2003, 2, 67.

(4) Yuriev, E.; Farrugia, W.; Scott, A. M.; Ramsland, P. A. Immunol. Cell Biol. 2005, 83, 709.

(5) Pérez, S.; Mouhous-Riou, N.; Nifant'ev, N. E.; Tsvetkov, Y. E.; Bachet, B.; Imberty, A. Glycobiology 1996, 6, 537. 
(6) Azurmendi, H. F.; Martin-Pastor, M.; Bush, C. A. Biopolymers 2002, 63, 89 .

(7) Battistel, M. D.; Azurmendi, H. F.; Frank, M.; Freedberg, D. I. J. Am. Chem. Soc. 2015.

(8) Cagas, P.; Bush, C. A. Biopolymers 1990, 30, 1123.

(9) Zierke, M.; Smiesko, M.; Rabbani, S.; Aeschbacher, T.; Cutting, B.; Allain, F. H.; Schubert, M.; Ernst, B. J. Am. Chem. Soc. 2013, 135, 13464.

(10) Homans, S. W.; Forster, M. Glycobiology 1992, 2, 143.

(11) Imberty, A.; Mikros, E.; Koca, J.; Mollicone, R.; Oriol, R.; Pérez, S. Glycoconj. J. 1995, 12, 331.

(12) McEver, R. P. Glycoconj. J. 1997, 14, 585.

(13) Pederson, K.; Mitchell, D. A.; Prestegard, J. H. Biochemistry 2014, 53,5700 .

(14)Scheffler, K.; Ernst, B.; Katopodis, A.; Magnani, J. L.; Wang, W. T.; Weisemann, R.; Peters, T. Angew. Chem. Int. Ed. Engl. 1995, 34, 1841.

(15) Haselhorst, T.; Weimar, T.; Peters, T. J. Am. Chem. Soc. 2001, 123, 10705 .

(16)Wimmerova, M.; Mitchell, E.; Sanchez, J. F.; Gautier, C.; Imberty, A. J. Biol. Chem. 2003, 278, 27059.

(17) Audfray, A.; Claudinon, J.; Abounit, S.; Ruvoën-Clouet, N.; Larson, G.; Smith, D. F.; Wimmerová, M.; Le Pendu, J.; Römer, W.; Varrot, A.; Imberty, A. J. Biol. Chem. 2012, 287, 4335.

(18)Topin, J.; Arnaud, J.; Sarkar, A.; Audfray, A.; Gillon, E.; Perez, S.; Jamet, H.; Varrot, A.; Imberty, A.; Thomas, A. PloS ONE 2013, 8, e71149.

(19) Houser, J.; Komarek, J.; Kostlanova, N.; Cioci, G.; Varrot, A.; Kerr, S. C.; Lahmann, M.; Balloy, V.; Fahy, J. V.; Chignard, M.; Imberty, A.; Wimmerova, M. PloS ONE 2013, 8, e83077.

(20) Ernst, B.; Magnani, J. L. Nat Rev Drug Discov 2009, 8, 661.

(21) Pérez, S.; Tubiana, T.; Imberty, A.; Baaden, M. Glycobiology 2015, $25,483$.

(22) Kostlanová, N.; Mitchell, E. P.; Lortat-Jacob, H.; Oscarson, S.; Lahmann, M.; Gilboa-Garber, N.; Chambat, G.; Wimmerová, M.; Imberty, A. J. Biol. Chem. 2005, 280, 27839.

(23) Kabsch, W. Acta Crystallogr. 2010, D66, 125.

(24)Winn, M. D.; Ballard, C. C.; Cowtan, K. D.; Dodson, E. J.; Emsley, P.; Evans, P. R.; Keegan, R. M.; Krissinel, E. B.; Leslie, A. G.; McCoy, A.; McNicholas, S. J.; Murshudov, G. N.; Pannu, N. S.; Potterton, E. A.; Powell, H. R.; Read, R. J.; Vagin, A.; Wilson, K. S. Acta Crystallogr. D. Biol. Crystallogr. 2011, 67, 235.

(25) McCoy, A. J.; Grosse-Kunstleve, R. W.; Adams, P. D.; Winn, M. D.; Storoni, L. C.; Read, R. J. J. Appl. Crystallogr. 2007, 40, 658.

(26) Langer, G.; Cohen, S. X.; Lamzin, V. S.; Perrakis, A. Nature Protocols 2008, 3, 1171.
(27)Murshudov, G. N.; Skubak, P.; Lebedev, A. A.; Pannu, N. S.; Steiner, R. A.; Nicholls, R. A.; Winn, M. D.; Long, F.; Vagin, A. A. Acta Crystallogr. D. Biol. Crystallogr. 2011, 67, 355.

(28) Emsley, P.; Lohkamp, B.; Scott, W.; Cowtan, K. Acta Crystallogr. D. Biol. Crystallogr. 2010, 66, 486.

(29) Keller, S.; Vargas, C.; Zhao, H.; Piszczek, G.; Brautigam, C. A.; Schuck, P. Anal. Chem. 2012, 84, 5066.

(30) Brautigam, C. A. Methods Enzymol. 2015, 562, 109.

(31) Case, D. A.; Darden, T. A.; Cheatham III, T. E.; Simmerling, C. L.; Wang, J.; Duke, R. E.; Luo, R.; Walker, R. C.; Zhang, W.; Merz, K. M.; Roberts, B.; Hayik, S.; Roitberg, A.; Seabra, G.; Swails, J.; Götz, A. W.; Kolossváry, I.; Wong, K. F.; Paesani, F.; Vanicek, J.; Wolf, R. M.; Liu, J.; Wu, X.; Brozell, S. R.; Steinbrecher, T.; Gohlke, H.; Cai, Q.; Ye, X.; Wang, J.; Hsieh, M.-J.; Cui, G.; Roe, D. R.; Mathews, D. H.; Seetin, M. G.; Salomon-Ferrer, R.; Sagui, C.; Babin, V.; Luchko, T.; Gusarov, S.; Kovalenko, A.; Kollman, P. A.; University of California: San Francisco, 2012.

(32) Lindorff-Larsen, K.; Piana, S.; Palmo, K.; Maragakis, P.; Klepeis, J.; Dror, R. O.; Shaw, D. E. Proteins 2010, 78, 1950.

(33) Kirschner, K. N.; Yongye, A. B.; Tschampel, S. M.; GonzalezOuteirino, J.; Daniels, C. R.; Foley, B. L.; Woods, R. J. J. Comput. Chem. 2008, 29, 622.

(34) Anandakrishnan, R.; Aguilar, B.; Onufriev, A. V. Nucleic Acids Res. 2012, 40, W537.

(35) Myers, J.; Grothaus, G.; Narayanan, S.; Onufriev, A. Proteins 2006, 63, 928

(36) Maier, J. A.; Martinez, C.; Kasavajhala, K.; Wickstrom, L.; Hauser, K. E.; Simmerling, C. Journal of Chemical Theory and Computation 2015, 11, 3696.

(37) Grossfield, A.;

http://membrane.urmc.rochester.edu/content/wham: 1992.

(38)Schlitter, J.; Engels, M.; Krüger, P. J. J. Mol. Graph. 1994, 12, 84.

(39) Houser, J.; Komarek, J.; Cioci, G.; Varrot, A.; Imberty, A.;

Wimmerova, M. Acta Crystallogr. 2015, D71, 442.

(40) Perez, S.; Sarkar, A.; Rivet, A.; Breton, C.; Imberty, A. Methods Mol. Biol. 2015, 1273, 241.

(41) Bérces, A.; Whitfield, D. M.; Nukada, T. Tetrahedron 2001, 57, 477.

(42)Sattelle, B. M.; Almond, A. Carbohydr. Res. 2014, 383, 34.

(43) Sattelle, B. M.; Shakeri, J.; Almond, A. Biomacromolecules 2013, 14, 1149 .

(44) Sattelle, B. M.; Almond, A. Glycobiology 2011, 21, 1651.

(45) Hudson, K. L.; Bartlett, G. J.; Diehl, R. C.; Agirre, J.; Gallagher, T.; Kiessling, L. L.; Woolfson, D. N. J. Am. Chem. Soc. 2015.

(46) Wimmerova, M.; Kozmon, S.; Necasova, I.; Mishra, S. K.; Komarek, J.; Koca, J. PLoS One 2012, 7, e46032.

TOC

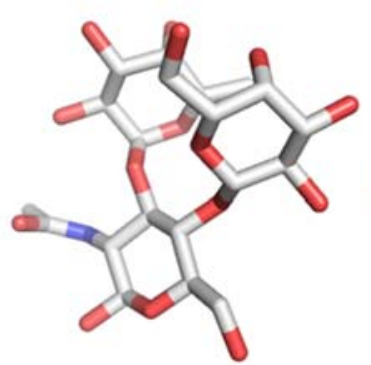

Closed

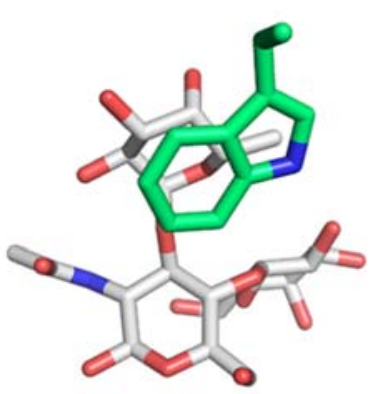

Open 